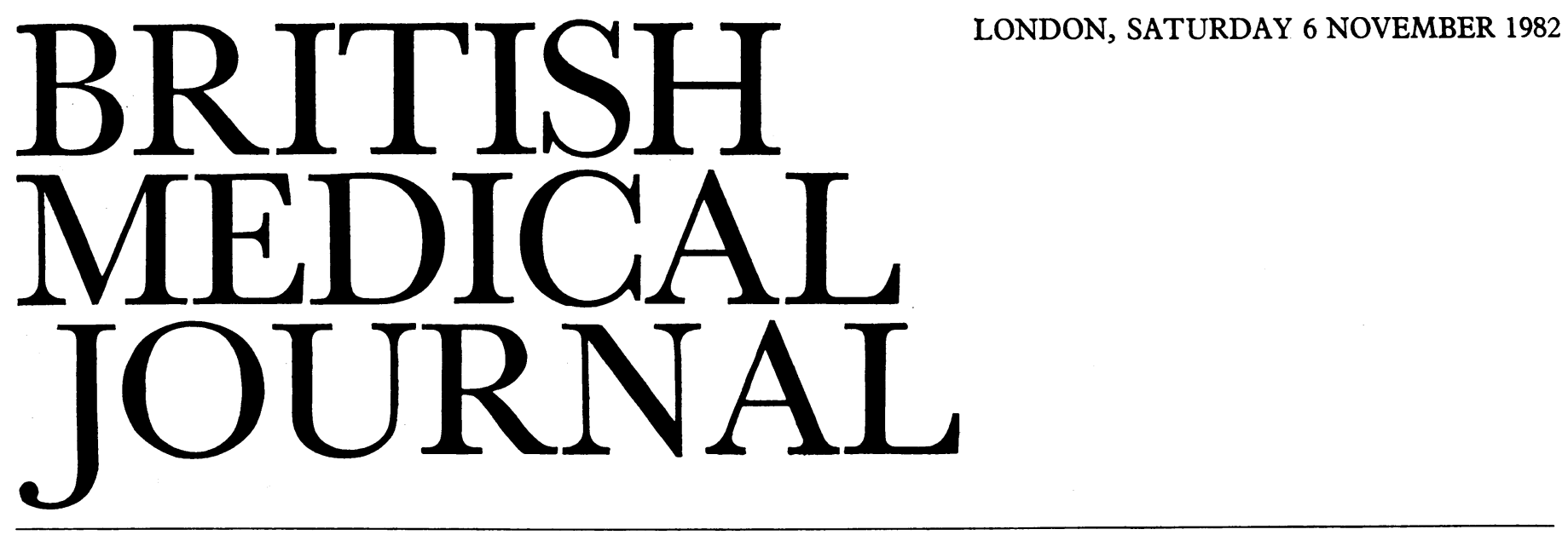

\title{
Managing hyperlipidaemias
}

The management of patients with hyperlipidaemias has long been controversial. The drugs that have been used, with varying biochemical effect, include clofibrate, nicotinic acid, Dthyroxine, and the ion-exchange resins cholestyramine and colestipol. Drug treatment has been criticised, however, on the grounds of doubts about its clinical benefits and also because of proved or anecdotal side effects. Two new drugs are being advertised widely and attractively, probucol for the treatment of hypercholesterolaemia and bezafibrate for that of all hyperlipidaemic disorders except lipoprotein lipase deficiency (type I in the World Health Organisation classification). The introduction of these new agents makes it timely to review the state of knowledge and opinion on which types of patients should be treated, what treatment might achieve, and which treatments are likely to be effective.

In most cases hyperlipidaemia is a biochemical diagnosis based on the presence of raised fasting plasma concentrations of cholesterol or triglyceride or both. Its classification has been reviewed elsewhere. ${ }^{1-3}$ The diagnosis demands careful attention to several rules for sampling, without which spurious results are likely to be obtained. ${ }^{2}$ Measurement of plasma lipids should be made only in specimens obtained after a 10-14hour fast. Before sampling, the patient should have conformed to his accustomed diet, alcohol consumption, and general life style for at least a fortnight, though only a minimum of alcohol should be taken on the day before sampling. A standardised procedure for venepuncture should be adopted-with, for example, the patient remaining seated for 15 minutes beforehand-and care should be taken to avoid prolonged venous stasis, which can increase plasma cholesterol and triglyceride concentrations by up to $10-20 \%$. If plasma is analysed within about three months of myocardial infarction, major surgery, or other serious illness the results are likely to be misleading because of the prolonged hypertriglyceridaemia, and sometimes depression of cholesterol concentrations, associated with these conditions. Even minor illnesses such as influenza may cause a transient depression of cholesterol concentrations and so mask a lipid disorder. Many patients have been subjected to the protracted expense and inconvenience of treatment for a spurious hyperlipidaemia while others have had their hypercholesterolaemia missed because of overzealous investigations too soon after myocardial infarction or vascular surgery.

Once a patient has been clearly shown to have hyperlipidaemia a search must be made for any underlying primary disease, the most common being diabetes mellitus and hypothyroidism. Other possible causes are renal failure and excess of endogenous or exogenous steroids. The patient should be asked about excessive consumption of alcohol, which is a particularly common cause of hyperlipidaemia, occasionally with a very severe rise in both cholesterol and triglyceride concentrations. Oestrogen-containing contraceptive pills may cause a rise in triglyceride values. True primary hyperlipidaemia frequently presents with a suggestive family history of early cardiovascular disease, but physical signs such as xanthomata or lipaemia retinalis are unusual, most cases being detected after biochemical screening tests on hospital patients or blood donors or by family studies.

Treatment of patients with hyperlipidaemias is still controversial. Probably, however, patients with hypercholesterolaemia do require active treatment. The risk of ischaemic heart disease increases progressively with serum low-density lipoprotein (LDL) cholesterol, an increase exacerbated by other factors such as smoking and hypertension. Lowering cholesterol concentrations in hypercholesterolaemic patients should therefore be of clinical benefit, though this view has not been fully supported by strictly controlled trials. Whether or not lipid-lowering drugs give any more benefits than those obtainable from proper dietary control remains unclear. The United States Lipid Research Clinics Programme is conducting a randomised double-blind trial over seven years comparing the effects of cholestyramine and placebo in men with type II hyperlipidaemia who are also taking cholesterol-lowering diets. ${ }^{4}$ The results of this trial, due in 1983, may answer the question. Though treatment of hypercholesterolaemia in primary prevention of cardiovascular disease may be effective, such treatment after myocardial infarction is unlikely to improve prognosis, as shown by the results of the Coronary Drug Project Trial. ${ }^{5}$ Few doctors, however, will neglect dietary advice for their hypercholesterolaemic patients after an infarction, though the use of lipid-lowering drugs is possibly not appropriate. Clearly risk factors such as hypertension and smoking need to be tackled at the same time as hypercholesterolaemia.

Patients with a mild to moderate rise in plasma triglyceride concentrations but with no hypercholesterolaemia are probably not at increased risk of cardiovascular disease and thus do not require vigorous treatment. High concentrations of triglycerides may, however, cause painful enlargement of the liver 
and spleen and may precipitate covert or overt pancreatitis. Such patients may also develop disfiguring and painful eruptive xanthomata, which are subject to ulceration, discharge, and infection. These patients need active treatment. Dietary restriction of fat, carbohydrate, and, in particular, alcohol will usually rapidly lower triglyceride concentrations with early resolution of visceral symptoms and regression of cutaneous lesions. There may be some justification for using lipid-lowering agents in the few patients with a persisting large rise in serum triglyceride concentrations despite dietary compliance and weight reduction.

These general principles provide a basis for an approach to the patient with proved hyperlipidaemia. The cornerstone must be the intention to treat the patient rather than his biochemical results. Firstly, and most important, the patient should be given detailed dietary advice with a target to aim at if overweight. Simply handing out leaflets and "calorie counters" is not enough. Most doctors have access to the skills of health service dietitians, either community based or in a local hospital, and they should use this service early.

Patients who fail to comply with diet sufficiently to achieve their optimum body weight are unlikely to respond satisfactorily to drug treatment. Prescription of drugs is certainly inappropriate in the first instance in hyperlipidaemia without first providing detailed dietary advice and making sure such advice is understood and followed. A year's treatment with lipid-lowering agents costs from about $£ 40$ for clofibrate to $£ 400$ for ion-exchange resins in minimum adult doses, while dietary treatment is generally both cheap and free of adverse effects.

Monitoring the treatment of patients with hyperlipidaemia requires assessment of clinical symptoms and signs, if any, body weight, and measurement of fasting plasma cholesterol and triglyceride concentrations. The plasma cholesterol concentration is not, however, a simple risk factor, since it includes the high-density lipoprotein (HDL) cholesterol, which is thought to exert a protective influence. ${ }^{6}$ The LDL: HDL ratio may be a more reliable risk factor, particularly if the $\mathrm{HDL}_{2}$ subfraction is measured. ${ }^{7}$ Thus the notion of a regimen or drug that is cholesterol lowering is simplistic, since in some instances both LDL cholesterol ("bad") and HDL cholesterol ("good") may be reduced. Furthermore, the real test of a treatment is the effect on the whole patient not just on biochemical changes in the plasma.

Patients will need referral to specialist clinics with an interest in lipid disorders when their response to diet is poor or the primary physician is unhappy about proceeding further on his own. Such clinics give further clinical assessment and advice from attending dietitians and can often provide extra psychological pressure to follow this advice. They also provide the opportunity for further investigation. Since the staff of lipid clinics see many patients with hyperlipidaemia and are familiar with current ideas they are in the best position to advise whether or not to start drug treatment.

The drugs used in the treatment of hyperlipidaemia have controversial clinical benefits and they are likely to be used for very long periods by patients. Ideally, therefore, they should be prescribed only in properly controlled trials designed to clarify their benefits and risks. Attention has recently been drawn to the finding of electrocardiographic abnormalities, particularly an increased $\mathrm{Q}-\mathrm{T}$ interval, in patients receiving probucol long term. ${ }^{8}$ What this means clinically in terms of real risk in man remains uncertain. ${ }^{9}$ The finding should serve, however, as a reminder that few drugs are entirely without risk, particularly if taken over long periods. Prescribing and taking drugs may seem simple but requires careful and $\underline{\underline{\sigma}}$ informed selection of those patients in whom benefits are likely to exceed possible risks.

Patients with hyperlipidaemia present important questions and opportunities in the primary prevention of major diseases. We will not answer these questions by treating disorders about which we know little with drugs which we are not sure are effective unless we organise such treatment to provide information. Drugs which are potentially important if used selectively will, unless we are careful, be submerged one by one by the same tide of criticism which has almost swamped clofibrate-a tide generated by excessive and inappropriate prescribing.

BRENDAN M BUCKLEY Senior registrar

ALAN M Bold

Consultant chemical pathologist

Lipid Clinic,

Clinical Chemistry Department,

Queen Elizabeth Hospital,

Birmingham B15 2TH

1 Beaumont JL, Carlson LA, Cooper GR, Fejfar Z, Fredrickson DS, Strasser T. Classification of hyperlipidaemias and hyperlipoproteinaemias. Bull WHO 1970;43:891-915.

2 Lewis B. The hyperlipidaemias: clinical and laboratory practice. London: Blackwell Scientific Publications, 1976.

${ }^{3}$ Chait A, Brunzell JD. Hyperlipidemias. Medicine International 1981 ; 1 : 359-63.

${ }^{4}$ Rifkind B, Boor R. The Lipid Research Clinics Coronary Primary Prevention Trial. In: Fumagalli R, Kritchevsky D, Paoletti R, eds. Drugs affecting lipid metabolism. Amsterdam: Elsevier/North Holland, 1980:151-7.

5 The Coronary Drug Project Research Group. Clofibrate and niacin in coronary heart disease. $\mathcal{F} A M A$ 1975;231:360-81.

${ }^{6}$ Miller GJ, Miller NE. Plasma-high-density-lipoprotein concentration and development of ischaemic heart-disease. Lancet $1975 ; \mathrm{i}: 16-9$.

${ }^{7}$ Miller NE, Hammett F, Saltissi S, et al. Relation of angiographically defined coronary artery disease to plasma lipoprotein subfractions and apolipoproteins. $\mathrm{Br}$ Med $\mathcal{F} 1981 ; 282: 1741-4$.

${ }^{8}$ Troendle G, Gueriguian J, Sobel S, Johnson M. Probucol and the QT interval. Lancet 1982;i:1179.

${ }^{9}$ Martz BL. Probucol and the QT interval. Lancet 1982 ;i:1365.

\section{Immunisation of infants at high risk of hepatitis $B$}

Hepatitis B virus is maintained in populations by the existence of a symptomless carrier state. Transmission from highly infective carrier mothers to their newborn by contamination with blood at birth helps to perpetuate the infection since neonates (probably because of the immaturity of their immune systems) usually respond by developing persistent symptomless carriage of hepatitis $B$ virus.

This type of transmission is common in areas with a high prevalence of carriage of hepatitis B virus in the populationfor example, south-east Asia, where the proportion is $10-20 \%$ -and rare where it is low-for example, north-west Europe, where it is $0 \cdot 1-0.5 \%$. The risk to the newborn is also influenced by ethnic differences in the infectivity of carriers. High prevalence and infectivity combine to present the greatest risk to Chinese infants, with a progressive decline in order of risk to the newborn in Africans, Asians, and Caucasians-in whom highly infective carriage of hepatitis B virus is rare. ${ }^{1}$

The infectivity of blood which contains hepatitis B surface antigen (HBsAg) can be gauged by serological tests. Blood containing hepatitis e surface antigen ( $\mathrm{HBeAg}$ ) is highly infective; blood which gives negative results for $\mathrm{HBeAg}$ and 